\title{
Infrared Signatures of the Dynamic Jahn-Teller Effect in Fullerene-Based Materials
}

\author{
K. Kamarás and G. Klupp \\ Research Institute for Solid State Physics and Optics, Hungarian Academy of Sciences, P.O. Box 49, H-1525 \\ Budapest, Hungary
}

\begin{abstract}
Temperature-dependent vibrational spectra show the evolution of dynamical Jahn-Teller states in $\mathrm{A}_{2} \mathrm{C}_{60}$ and $\mathrm{A}_{4} \mathrm{C}_{60}$ fulleride salts. Whereas at low temperature the external field of the cations determines the local symmetry, at high temperature the fullerene balls distort into a $\mathrm{D}_{3 d}$ or $\mathrm{D}_{5 d}$ symmetry, independent of the surrounding lattice. The average structure is preserved while the molecules show pseudorotation between possible potential minima.
\end{abstract}

Keywords: fullerenes, infrared spectroscopy, Jahn-Teller effect

PACS: $71.70 \mathrm{Ej}, 78.30+-\mathrm{j}, 78.30 \mathrm{Na}$

\section{INTRODUCTION}

Alkali salts of the fullerene $\mathrm{C}_{60}$ with an even number ( 2 or 4 ) of electrons have been a subject of controversy since the discovery of this family of materials. Bandstructure calculations predicted them to be metals, but experiments found them to be insulators. The theoretical explanation for this behavior, formulated by Fabrizio and Tosatti [1], is the Mott-Jahn-Teller nonmagnetic insulating state. In this picture, the molecules undergo a JahnTeller distortion and at the same time, a Mott localization, yielding narrow bands separated by the Jahn-Teller splitting. Possible geometries, derived from group theoretical calculations [2], are $\mathrm{D}_{3 d}$ and $\mathrm{D}_{5 d}$ in the absence of an external potential and $\mathrm{D}_{2 h}$ when an appropriate external potential is present. Such a potential can be caused by the surrounding cations and, in both orthorhombic and tetragonal crystals, is compatible with the $\mathrm{D}_{2 h}$ symmetry.

Several experimental findings, both in isolated ions in solution [3] and in the solid state [4-6], have been explained by a Jahn-Teller distortion. However, the question remains hitherto unanswered how much the external crystal field influences the electronic and vibrational levels and how much the internal degrees of freedom contribute.

In the simplest approximation where the fullerene molecule is considered spherical (a "giant atom") [5, 7], the symmetry of the crystal is taken as the primary perturbation. In a tetragonal field the sphere distorts to a spheroid and the energy levels show a twofold splitting (Table I). If the symmetry is lowered to orthorhombic, the splitting increases threefold. However, if we start from an icosahedral symmetry, as must be the case for molecular vibrations with the motion of atoms on the apices of the $\mathrm{C}_{60}$ structure taken into account, then we have to consider the molecular symmetry instead of the
TABLE 1. Expected level splittings of fullerene levels in different environments

\begin{tabular}{lcccc}
\hline & Isolated & fcc & bet & bco \\
\hline \multirow{2}{*}{ sphere $\left(\mathrm{p}^{3}\right)$} & --- & --- & -- & - \\
& & & & - \\
\hline \multirow{2}{*}{ icosahedron } & --- & --- & - & - \\
& & & - & - \\
\hline \multirow{2}{*}{ vibrations } & --- & --- & - & - \\
& & & - & - \\
\hline point group & $\mathrm{I}_{h}$ & $\mathrm{~T}_{h}$ & $\mathrm{D}_{2 h}$ & $\mathrm{D}_{2 h}$ \\
\hline
\end{tabular}

simplified picture outlined above. (The transition from the spherical approximation to the more realistic icosahedral model is called "warping" and it can be neglected in some models of electronic structure [2], but certainly not when motions of the nuclei are involved.)

$\mathrm{A}_{4} \mathrm{C}_{60}$ salts are modeled as the superposition of two standard orientations [8], each having a $\mathrm{D}_{2 h}$ symmetry. This would mean that in both orthorhombic and tetragonal crystals, the vibrational levels should show a threefold splitting. This is, however, not what Iwasa and Kaneyasu [4] have observed. At room temperature they saw a twofold splitting. Our previous infrared measurements [9] found this twofold splitting changing to a threefold one at low temperatures, while the crystal structure remained tetragonal [10]. In this paper, we compare the vibrational spectra of $\mathrm{A}_{2} \mathrm{C}_{60}$ and $\mathrm{A}_{4} \mathrm{C}_{60}$ salts in various crystal structures and their temperature dependences, in order to establish the factors influencing the symmetry of the molecular ions. 


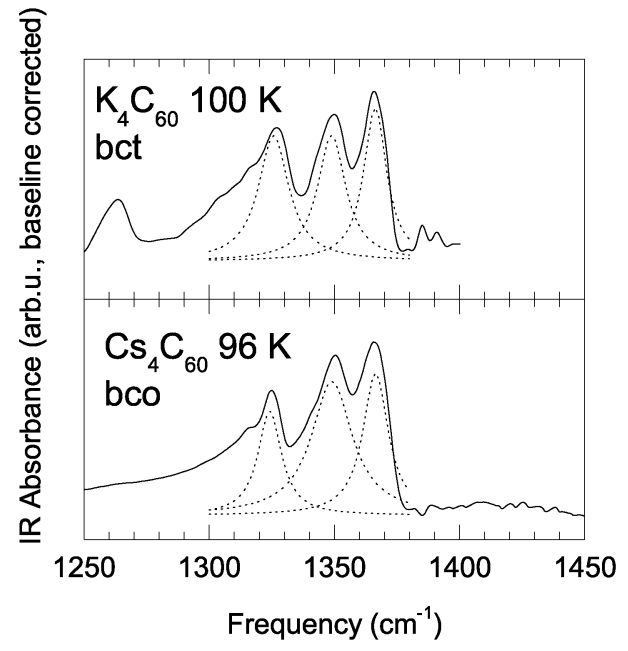

FIGURE 1. Low-temperature infrared spectra of $\mathrm{A}_{4} \mathrm{C}_{60}$ salts $\left(\mathrm{D}_{2 h}\right.$ symmetry).

\section{EXPERIMENTAL}

Fullerene salts were prepared by solid-state synthesis from $\mathrm{C}_{60}$ and the respective alkali metals. As the materials are sensitive to oxygen, the preparation and all subsequent handling was performed in an inert atmosphere. Infrared spectra were taken by a Bruker IFS 28 FTIR instrument with a $2 \mathrm{~cm}^{-1}$ resolution.

\section{RESULTS AND DISCUSSION}

In the two salts studied at low temperature (Figure 1), the predictions of Table I are met: in both orthorhombic and tetragonal environments, the spectra are consistent with a $\mathrm{D}_{2 h}$ molecular symmetry.

In $\mathrm{Cs}_{4} \mathrm{C}_{60}$, on warming a structural phase transition occurs from orthorhombic to tetragonal [11], but in $\mathrm{K}_{4} \mathrm{C}_{60}$ no such transition was seen [10]. The vibrational spectra, however, change in both cases (Figure 2) to a pattern corresponding to a $\mathrm{D}_{3 d} / \mathrm{D}_{5 d}$ symmetry. The same splitting is seen in $\mathrm{Na}_{2} \mathrm{C}_{60}$ at high temperature, where the crystal structure is face-centered cubic [12]. (At low temperature, this latter salt undergoes a segregation into nanodomains of $\mathrm{C}_{60}$ and $\mathrm{Na}_{3} \mathrm{C}_{60}$ [13]; thus the spectrum is not comparable to that of the other alkali salts.) The position of the center of the structure corresponds to the empirical relation between the molecular charge and the frequency of the $\mathrm{T}_{1 u}(4)$ mode [14].

From these results we can conclude that temperature, rather than external potential, is the driving force behind the spectral changes in the alkali fulleride salts. With in-

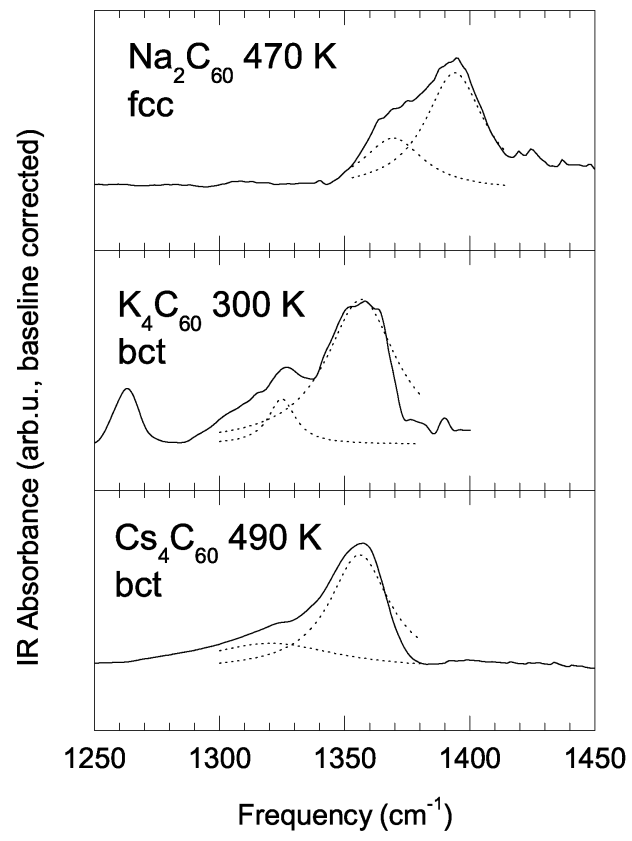

FIGURE 2. High-temperature infrared spectra of $\mathrm{A}_{n} \mathrm{C}_{60}$ salts ( $\mathrm{n}=2,4, \mathrm{D}_{3 d} / \mathrm{D}_{5 d}$ symmetry). The position of the peak reflects the charge transfer on the fullerene ball.

creasing temperature, the thermal motion of the fullerene balls intensifies. One such typical motion is pseudorotation, by which the molecule can distort from its lowtemperature shape and assume a principal $\mathrm{C}_{3}$ axis in the center of a hexagon or a $\mathrm{C}_{5}$ axis in the center of a pentagon. The preferred axis can point in many possible directions, and an isolated fullerene ball can perform continous pseudorotation between these distortions. Calculations show [2] that the $\mathrm{D}_{3 d} / \mathrm{D}_{5 d}$ shapes are minima on the adiabatic potential energy surface (APES) and the $\mathrm{D}_{2 h}$ shapes are saddle points for isolated ions. Thus the most probable dynamics is tunneling between the $\mathrm{D}_{3 d} / \mathrm{D}_{5 d}$ states while avoiding the $\mathrm{D}_{2 h}$ states.

Deviations from the atomic positions in the icosahedral symmetry are very small, of the order of 0.05 Angstroms [3, 8]. Because of the minuteness of the distortion in question, and because of the dynamic nature of the high-temperature state, X-ray diffraction does not detect any deviation. While the average atomic positions, detectable by X-ray diffraction, do not change, the average molecular symmetry does; therefore, vibrational spectroscopy is a more sensitive indicator of the dynamical Jahn-Teller effect than diffraction.

Pseudorotation should not be confused with molecular reorientation, whereby the fullerene ball rotates from one standard orientation to the other. The impor- 
tance of the $\mathrm{C}_{3}$ axes in this reorientation process has been emphasized before [15]. Reorientation happens on a much slower time scale than pseudorotation and does not change the symmetry; therefore we do not expect it to have any effect on the vibrational spectra. These two kinds of motions, though, might be the "fast" and "slow" rotations observed in NMR measurements [16-18].

\section{CONCLUSIONS}

From the temperature dependence of the infrared spectra of the $\mathrm{A}_{n} \mathrm{C}_{60}(\mathrm{n}=2,4)$ fulleride salts we have shown that these materials are true Mott-Jahn-Teller insulators. The molecular symmetry of the fullerene balls is determined by the external potential caused by the surrounding cations at low temperature, and by the molecular Jahn-Teller distortion at high temperature.

\section{ACKNOWLEDGMENTS}

We thank G. Oszlányi for invaluable help with X-ray diffraction measurements and useful discussions. This work was supported by the Hungarian National Research Fund through OTKA grant no. 049338.

\section{REFERENCES}

1. M. Fabrizio and E. Tosatti, Phys. Rev. B 55, 13465 (1997).

2. C. C. Chancey and M. C. M. O'Brien, The Jahn-Teller Effect in $C_{60}$ and Other Icosahedral Complexes, Princeton University Press, Princeton, 1997.

3. C. A. Reed and R. D. Bolskar, Chem. Rev. 100, 1075 (2000).

4. Y. Iwasa and T. Kaneyasu, Phys. Rev. B 51, 3678 (1995).

5. R. Kerkoud, P. Auban-Senzier, D. Jérome, S. Brazovskii, N. Kirova, I. Luk'yanchuk, F. Rachdi, and C. Goze, Synth. Met. 77, 205 (1996).

6. M. Knupfer and J. Fink, Phys. Rev. Lett. 79, 2714 (1997).

7. L. Forró and L. Mihály, Rep. Progr. Phys. 64, 649 (2001).

8. C. A. Kuntscher, G. M. Bendele, and P. W. Stephens, Phys. Rev. B 55, R3366 (1997).

9. K. Kamarás, G. Klupp, D. B. Tanner, A. F. Hebard, N. M. Nemes, and J. E. Fischer, Phys. Rev. B 65, 052103 (2002).

10. G. Klupp, K. Kamarás, N. M. Nemes, C. M. Brown, and J. Leao, AIP Conference Proceedings 723, 8 (2004).

11. P. Dahlke and M. J. Rosseinsky, Chem. Mater. 14, 1285 (2002).

12. T. Yildirim, J. E. Fischer, P. W. Stephens, and A. R. McGhie, in Progress in Fullerene Research, edited by H. Kuzmany, J. Fink, M. Mehring, and S. Roth (World Scientific, Singapore, 1994), p. 235.

13. G. Klupp, K. Kamarás, N. M. Nemes, P. Matus, D. Quintavalle, L. F. Kiss, É. Kováts, S. Pekker, and A. Jánossy, AIP Conference Proceedings, in press.

14. T. Pichler, R. Winkler, and H. Kuzmany, Phys. Rev. B 49, 15879 (1994).
15. D. A. Neumann, J. R. D. Copley, D. Reznik, W. A. Kamitakahara, J. J. Rush, R. L. Paul, R. M. Lindstrom, J. Phys. Chem. Solids 54, 1699 (1993).

16. G. Zimmer, M. Helmle, M. Mehring, and F. Rachdi, Europhys. Lett. 27, 543 (1994).

17. G. Zimmer, M. Mehring, C. Goze, and F. Rachdi, Phys. Rev. B 52, 13300 (1995).

18. V. Brouet, H. Alloul, S. Garaj, and L. Forró, Phys. Rev. $B$ 66, $155122(2002)$ 